\title{
A miniaturised active thermography system to inspect composite laminates
}

\author{
Weixiang Du, Haochen Liu, Yitian Zhao, Adisorn Sirikham, Sri Addepalli, and Yifan Zhao*
}

\begin{abstract}
With the rapid increase of the integration and complexity of industrial components, the inaccessibility and inapplicability of existing Non-destructive testing devices have become a bottleneck for in-situ inspection of these objects. This paper introduces a miniaturised active thermography system featured with a small size, low resolution and low-cost thermal sensor, where two optional excitation sources including flash and laser are integrated. Dedicated data analysis approaches to evaluate defects are proposed considering the degraded signal quality. Three carbon fibre reinforced polymer laminates with a variety of defects are evaluated quantitatively and qualitatively using the proposed system by comparing with two existing nonminiaturised inspection systems. The results show that the proposed system can work effectively for the degradation assessment of composite laminates. Even with the technical limitations that affect the detectability, for instance, the low pixel resolution, this technique will play an important role to inspect components featured with geometrically intricate space.
\end{abstract}

Index Terms - NDT; active thermography; miniaturisation; degradation assessment; composite materials; in-situ inspection

\section{INTRODUCTION}

$\mathrm{T}$ HE damage of key components can endanger the overall safety and integrity of the engineering structures. Nondestructive testing (NDT) is an attractive technique for detecting the defects or material non-uniformity without damaging and undermining the targeted components or systems. However, with the increasing integration level and complexity of industrial systems, the size, weight and volume of NDT equipment become a bottleneck to improve the accessibility and applicability of in-situ inspection systems. It is particularly problematic for the challenging environments, such as systems with geometrically intricate space (e.g. aero-engines), hazardous condition (e.g. nuclear power generation) in some safety-critical industrial sectors, high-altitude and difficult-toclimb targets (e.g. wind turbine blades), highly constrained and semi-closed environment (e.g. aircraft wing box), where it is frequently impossible to use "off-the-shelf" equipment for insitu testing. Moreover, the serviceability of the components or systems to be inspected is a huge challenge, particularly in

Manuscript submitted on July 06, 2020; revised on September 5, 2020 and September 30, 2020; accepted on October 03, 2020.

W. Du, H. Liu, S. Addepalli, and Yifan Zhao are with the School of Aerospace, Transport and Manufacturing, Cranfield University, College Road, Cranfield, Bedfordshire, MK43 0AL, UK. determining their maintenance requirements as disassembly and inspection can be very time-consuming and expensive. Therefore, it is strongly demanded to develop lightweight and miniaturised NDT systems that can be deployed by miniaturised robotic systems [1], [2].

Among the various NDT techniques, visual and optical testing have the characteristics of being intuitive, non-contact and robust. Industrial endoscopes armed with mini fibre optic probes [3] can extend the inspector's sight distance and have the ability to inspect hard to reach and or inaccessible areas, but they are limited to detect small surface defects and cannot be used for sub-surface inspection. Options such as the Miniature Fiberscope [4], which is a representative endoscope produced by Karl Storz featuring a very small diameter of between $0.35 \mathrm{~mm}$ and $1.3 \mathrm{~mm}$, do exist in the market. Alternate techniques such as the magnetic particle inspection [5] is suitable for the inspection of ferromagnetic materials, but the lift force of the yoke and defect detection rate will be reduced when the volume of the device decreases. The permanent magnet can be made small enough to fit into tight areas, but the lift force is difficult to control. For instance, the RPNSS and RPNSL permanent magnets equipment manufactured by Johnson \& Allen Company are relatively small, but the lifting force is limited to $18 \mathrm{~kg}$ [6]. Radiographic testing can achieve accurate inspection for sub-surface defects using bulky computerised tomography (CT), such as Versatile Industrial CT Scanner [7]. Although there is evidence that a combination of $\mathrm{X}$-ray machine and film can be used for in-situ inspection [8], including the upgraded equipment like DXR250C-W Wireless Digital X-ray Detector produced by GE [9], miniaturisation of radiographic inspection is still a challenge due to the influence of radioactivity and its strict requirements on the detection environment. Portable and pocket-type ultrasonic testing equipment [10] has become more and more popular. A typical example is the ISONIC utPod produced by Sonotron NDT, which is an ultra-portable and multi-purpose ultrasonic testing instrument and weighs at $400 \mathrm{~g}$ [11]. However, the mandatory requirement of a coupling agent and the blind regions on the surface, limit its application for thin and complex-shaped workpieces with additional issues coming from cramped and inaccessible spaces [12]. Responding to the increasing demand for miniaturisation, the above features limit the progress and

Yitian Zhao is with the Cixi Institute of Biomedical Engineering, Ningbo Institute of Materials Technology and Engineering, Chinese Academy of Sciences, Ningbo, China.

A. Sirikham is with the Electronics and Telecommunication Engineering with Rajamangala University of Technology Krungthep, Bangkok, Thailand.

*Corresponding author: Yifan Zhao (e-mail: yifan.zhao@ cranfield.ac.uk). 


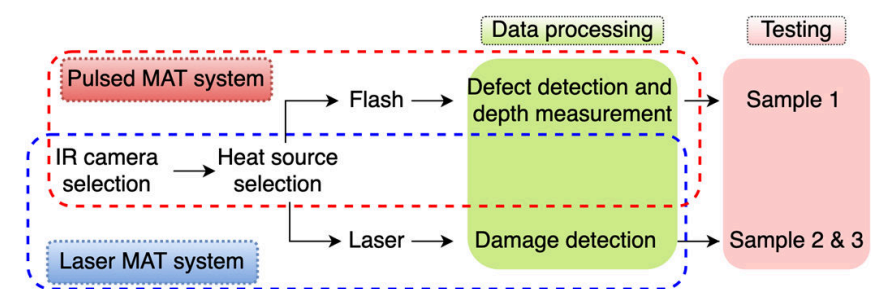

Fig. 1. The proposed methodology of this study.

applications of corresponding NDT techniques in the context of in-situ inspection.

As a powerful NDT technique, infrared thermography can provide rapid, non-contact, and robust non-invasive detection of both surface and sub-surface defects/damage. However, at present, the existing miniaturised infrared cameras are mostly used in passive thermography, with a few studies investigating its application in the active thermographic inspection [13][14][15]. Fuente et al. [16] used the reflection of the mirror to integrate the IR camera and laser into the borescope for pipe inspection. Du et al. [17] used the super-resolution method to improve the thermal image quality of low-resolution IR cameras. Even for these studies, the volume of integrated equipment available for industrial composite inspection is still relatively large. This paper reports a novel miniaturised active thermography (MAT) system with its qualitative and quantitative performance evaluation of a variety of defects in composites using two types of excitation: flash and laser, respectively. Particularly, for the laser MAT system, a unique inspection platform using an automatic XY stage is introduced, which has the potential for in-line inspection. Current miniaturised IR cameras feature relatively low-resolution and low signal-to-noise-ratio (SNR), an effective image processing and feature extraction method is therefore introduced to reduce the influence of noise and enhance the detectability of the damage. Additionally, two high-resolution thermal cameras are employed to validate the proposed MAT system.

\section{METHODS}

\section{A. Methodology}

The implemented methodology of this study can be illustrated by Fig. 1, which starts from the selection of sensor and excitation and follows data analysis methods for laser and flash MAT systems respectively. The proposed systems are then tested by three samples featuring a variety of defects.

For active thermography, the excitation sources usually can be divided into three categories: optical, mechanical and induction excitation [18]. Additionally, hot or cold gas/liquids as heat source has been revamped recently [19][20]. Both flash and laser belong to optical excitation, while ultrasonic belongs to mechanical excitation [21], and Eddy current is a typical representative of induction excitation [22]. The flash has the advantages of covering large excitation area with fast speed, but due to the factors such as irradiation distance and energy attenuation, the working distance should not be too far. Compared with the flash, the laser has advantages in energy density, intensity, accuracy, and ease in modulation[23]. It is an ideal excitation source for remote detection of delamination or crack defects in composites. Comparing with the Eddy current induction, the attenuation of the laser beam is very low, which leads to long-distance heating capacity and all these features
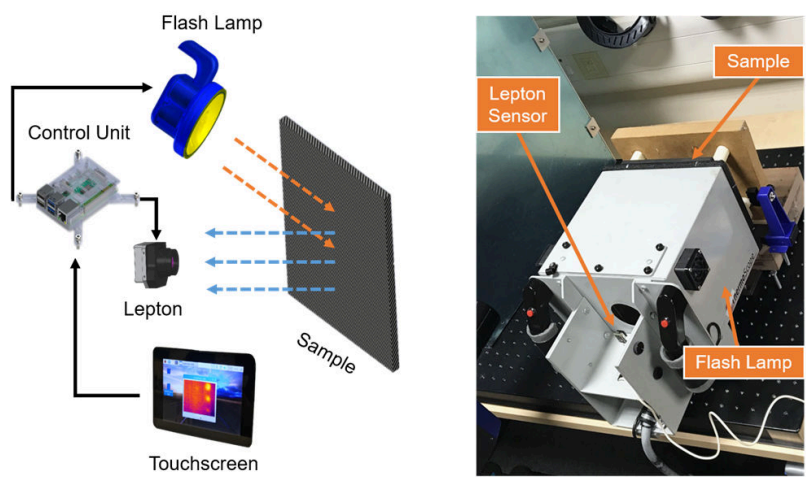

(a)
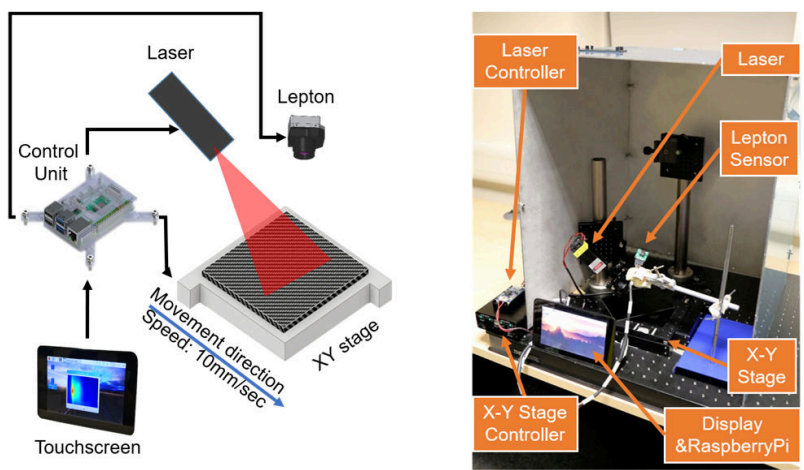

(b)

Fig. 2. The proposed two miniaturised active thermography systems. (a) MAT system 1 (excitation source: flash lamp); (b) MAT system 2 (excitation source: laser) (Note: the system is a fully enclosed system and at the time of this image, the system was turned off.)

support its application in a geometrically intricate environment. From the sensor point of view, due to the high-quality quantum detectors used in infrared cameras, especially the indium antimonide versions, the price of infrared cameras along with their relatively large size restricts their deployment for in-situ inspections. Considering large scale of tasks in in-situ inspection, low-cost and small-size infrared cameras are attractive for the stakeholders and inspection environment, but they usually compromise in spatial resolution, noise resistance, thermal sensitivity and sampling rate.

\section{B. MAT System}

The proposed MAT system consists of a miniaturised commercial infrared (IR) sensor, FLIR Lepton, and an external excitation source (either flash lamp or laser), all of which are integrated with a Raspberry Pi 3B+ along with a 7-inch touch screen (as shown in Fig. 2). The system works under the reflective mode, where the sensor and excitation source are on the same side of the targeted component. Comparing with other IR cameras commonly used in active thermography, such as FLIR SC7600 [24] and FLIR A655sc [25], Lepton has a much smaller size (similar to the UK's 5 pence coin). Table S1 in Supplementary Materials shows the comparison of the specifications of the FLIR SC7600, the A655sc and the Lepton, which will be tested and compared in this paper. The SC7600 has 4 times higher spatial resolution than the Lepton but is much bulky and weighs at almost 5000g in comparison with the $1 \mathrm{~g}$ weight of the Lepton. The cost of the SC7600 is hundred times 


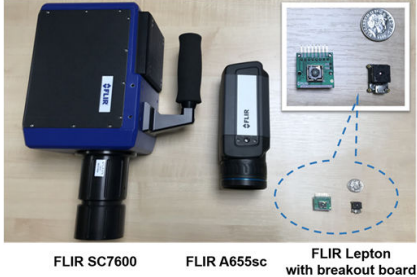

(a)

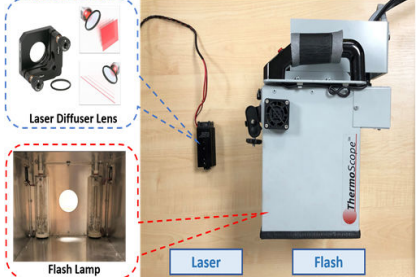

(b)
Fig. 3. (a) Comparison of the FLIR lepton with SC7600 and A655sc for active thermography. (b) Comparison of the laser and flash excitation sources

higher than that of the Lepton. The SC7600 has a better Noise Equivalent Temperature Difference (NETD) and frame rate. Considering the fact that the Lepton has the maximum sample rate equal to $8.7 \mathrm{~Hz}$, this paper only tests its performance on composites that have relatively low thermal conductivity.

The proposed MAT system accommodates two types of excitation source: optical flash (Fig. 2(a)) and laser (Fig. 2(b)). The compatibility of multiple excitation sources enables the flexibility to detect a variety of defects. Flash and laser are not combined but chosen separately according to the characteristics of their different excitation modes to increase the flexibility of the MAT system for detecting different types of defect. For example, the flash excitation is suitable for large area defect such as delamination while the laser usually achieves good detection results for cracks or small damage. The pulsed MAT system is powered by two capacitor-bank-controlled xenon flash lamps assembled inside a box with internal reflectors with a nominal flash power output of $2 \mathrm{KJ}$ over a $250 \times 200 \mathrm{~mm}$ area (see Fig. 2(b)). The flash energy can be adjusted considering the adjustable working distance between $200 \mathrm{~mm}$ to $300 \mathrm{~mm}$. The laser MAT system employs a low-cost semiconductor laser generator with a $450 \mathrm{~nm}$ wavelength, and the laser head dimension of $86 \times 33 \times 33 \mathrm{~mm}$ (see Fig. 3(b)). The small size, directionality and flexibility of laser pattern allows the proposed MAT system working under geometrically intricate space. The $15 \mathrm{~W}$ laser beam is shaped to a line using an optical shaping lens (see Fig. 2(b)). Whilst the flash lamps provide superior inspection results both qualitatively and quantitatively, the ability to introduce such systems for in-accessible areas remains a challenge. The laser system, on the contrary, due to its compact design, non-attenuated energy exposure and the ability to reconstruct line-base data in real-time comes as an alternate technique that shows great potential for such intricate inspections.

Dedicated algorithms and corresponding software implementation were developed and integrated with the Raspberry Pi to capture, store and process the data. Though the computing capability is not as powerful as traditional PCs, the computational time is acceptable considering the relatively small size of IR images and low sample rate. The raw thermal images and results of the analysis can be visualised by a mini touch screen.

\section{Data processing}

One objective of this study is to identify and develop appropriate quantitative evaluation algorithms for this MAT system, specifically considering the fact the sensor has a significantly low SNR. This section introduces a few methods for defect enhancement and depth measurement, for laser thermography and pulsed thermography respectively.

1) Pulsed thermography

Based on the Fourier heat transfer law, the surface temperature due to a defect at depth $L$ for a plate is given by [26]

$$
T(t)=\frac{Q}{\sqrt{\pi \rho c k t}}\left[1+2 \sum_{n=1}^{\infty} \exp \left(-\frac{n^{2} L^{2}}{\alpha t}\right)\right]
$$

where $T(t)$ is the temperature variation of the surface at time $t$, $Q$ is the pulse energy, $\alpha$ is the thermal diffusivity of the material, $\rho, c$, and $k$ are the material density, specific heat capacity and thermal conductivity respectively.

For the pulsed MAT system, we propose to use the Logarithmic Second-Derivative method (LSD) [27] and the New Least-squares Fitting (NLSF) method [28] to estimate the defect depth. In LSD, the temperature decay curve is converted into the logarithmic domain, and then the curve is fitted with a polynomial model to reduce temporal noise and save storage space. This process is referred to as the Thermographic Signal Reconstruction (TSR) [27]. The peak of the second derivative of the TSR fitting is often used to estimate the defect depth. The polynomial model is written as

$$
\ln (T(t)) \approx \sum_{n=0}^{N} a_{n}(\ln (t))^{n}
$$

where $N$ is the order of the polynomial function (normally $N$ is chosen between 6 to 8), and $a_{n}$ are polynomial coefficients to be estimated. The first and second derivative of TSR can also be used for defect enhancement [29] by providing higher defect contrasts. It can be computed by

$$
\begin{gathered}
\frac{d \ln (T(t))}{d \ln (t)} \approx \sum_{n=1}^{N} a_{n} \cdot n \cdot(\ln (t))^{n-1} \\
\frac{d^{2} \ln (T(t))}{d^{2} \ln (t)} \approx \sum_{n=2}^{N} a_{n} \cdot n \cdot(n-1) \cdot(\ln (t))^{n-2}
\end{gathered}
$$

If the thermal diffusivity $\alpha$ is known, the depth of defect can then be estimated by

$$
L=\sqrt{t_{L S D} \cdot \pi \cdot \alpha}
$$

where $t_{L S D}$ is the peak time of the second derivative.

The Least-Squares Fitting (LSF) method [30] uses a curvefitting approach based on a 1-D heat transfer model to fit the raw temperature decay curve to identify the defect depth. The model is written as

$$
T(t) \approx \frac{B}{\sqrt{t}}\left[1+2 \sum_{n=1}^{\infty} \exp \left(-\frac{n^{2} L^{2}}{\alpha t}\right)\right]-s t
$$

where $L$ is the sample or the defect depth and $B$ is a numerical value. This method has good resistance to noise, but it assumes a thermal wave reflection coefficient $(R)$ of 1 , which is not valid in most real situations. The NLSF method estimates the value of $R$ directly from the observation data and has a higher accuracy of defect depth estimation [28]. The analytical model of NLSF is written as

$$
\tilde{T}\left(t, A, W, R, t_{s}, s\right)=\frac{A}{\sqrt{t+t_{s}}}\left[1+2 \sum_{n=1}^{M} R^{n} \exp \left(-\frac{n^{2} W}{t+t_{s}}\right)\right]-s\left(t+t_{s}\right)
$$

where $A=\frac{Q}{\sqrt{\pi \rho c k}}, W=\frac{L^{2}}{\alpha}, t_{s}$ is the starting time of sampling, $s$ is 


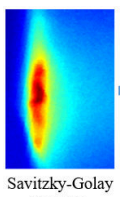
filtering

(a)

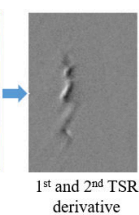

(b)

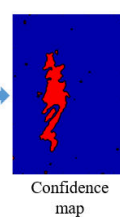

(c)

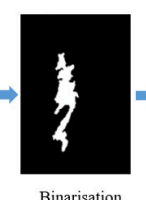

(d)

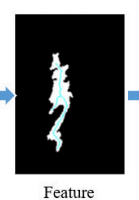

extractio

(e)

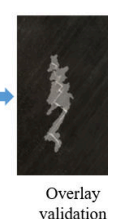

(f)
Fig. 4. The proposed damage evaluation process for the laser MAT system

the slope, and $M$ is a large iteration number. There are five parameters to be estimated including $A, W, R, t_{s}$, and $s$. A nonlinear least-squares solver in MATLAB (lsqnonlin) is applied to solve this five-parameter optimisation problem. The lower and upper bounds of $W$ and $A$ are usually selected as 5 times lower and 5 times higher than the initial values. The lower and upper bounds of $s$ are selected as -50 and 50, and the initial value is chosen as 0 . It should be noted that the computational time of this method depends on the selection of initial value and lower and upper bounds. Once the optimal parameters are estimated, if $\alpha$ is known, the defect depth can be estimated by

\section{2) Laser thermography}

$$
L=\sqrt{W \cdot \alpha}
$$

This paper proposes to use the Savitzky-Golay [31] filter, a digital smoothing filter, to increase the SNR of the low resolution and noisy IR images from Lepton. This is achieved by fitting successive time frames (or within a time window) with a pre-defined degree polynomial. As in the case of scattered signals, when the data points are equally spaced, an analytical solution can be derived in the form of a single set of convolution coefficients. The imaging of the $1^{\text {st }}$ and the $2^{\text {nd }}$ TSR derivatives of the filtered IR images can further reduce noise and enhance the true defect.

It should be noted that, in this study, the relative position between the laser and IR camera is fixed and the laser line is always on. In the raw IR images, the intensity of the laser line is so high that the damage contrast is very low and sometimes the damage is almost undetectable. The $1^{\text {st }}$ or $2^{\text {nd }}$ derivative between two adjacent frames will remove the laser line and preserve the change between these two images, by which means the contrast of defects/damage is significantly enhanced. The proposed process to evaluate damage using the laser MAT system can be illustrated by Fig. 4. Initially, the Savitzky-Golay filter is applied to the raw data cube to reduce temporal noise for all pixels (Fig. 4a). Then the process to calculate the $1^{\text {st }}$ or $2^{\text {nd }}$ TSR derivative using Eq. (3)-(4) is followed to remove the interference of the laser line and further reduce the temporal noise (Fig. 4b). A confidence map method [32] is applied to highlight the area with a significant difference from sound areas (Fig. 4c), where the intensity of thermal images is transferred into the confidence level of the inspection. By selecting an appropriate threshold (95\% confidence level is selected for this study), a binary image (Fig. 4d) is produced to isolate the damage. A skeleton extraction method is then used to describe the morphological feature of the detected damage for easy quantitative comparison and validation based on the overlay with the binary image (Fig. $4 \mathrm{e}$ ) or the $1^{\text {st }}$ or $2^{\text {nd }}$ TSR derivative image (Fig. 3f).

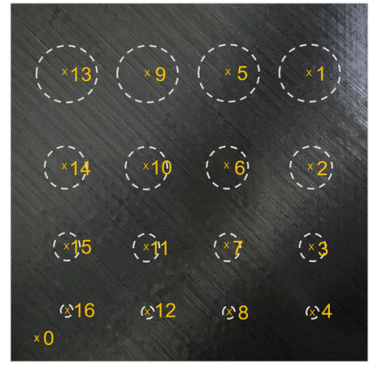

(a) Front side

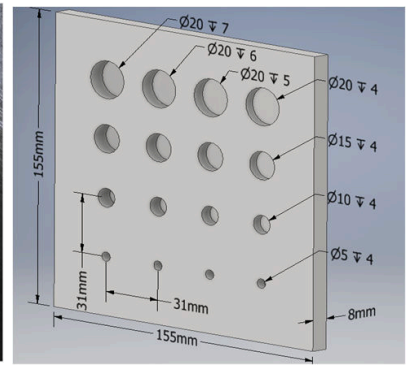

(b) Back side (3D view)
Fig. 5. Illustration of Sample 1 (CFRP). (a) Dash circle indicates the dimensions and locations of defects on the backside. Point 1-16 mark different defects and Point 0 is sampled from a sound region (reference region) (b) Design dimensions of defects on the backside.

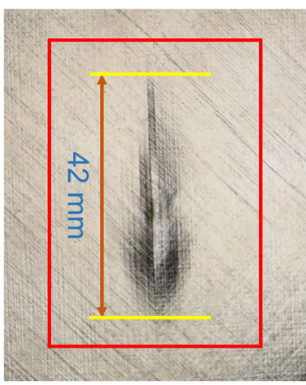

(a) Sample 2

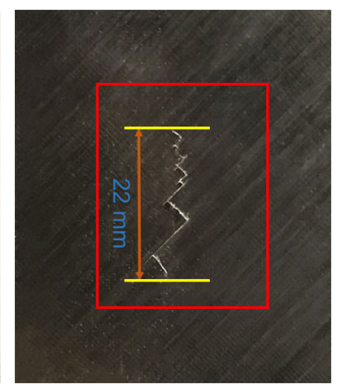

(b) Sample 3
Fig. 6. Illustration of the inspection surface of Sample 2 and 3, where delamination and crack are presented.

\section{Experimental Evaluation}

\section{1) Samples}

This paper employs three Carbon Fibre Reinforced Plastics (CFRP) laminates with different types of defect, marked as Sample 1, Sample 2 and Sample 3. All CFRP laminates were made by unidirectional Toray 800 carbon fibres preimpregnated with Hexcel M21 epoxy resin. Sample 1 has a dimension of $155 \times 155 \times 8 \mathrm{~mm}^{\wedge} 3$ and contains 16 artificial flatbottomed defects. The specification of Sample 1 and a snapshot are shown in Fig. 5. Point 1-16 indicate different defects and Point 0 is sampled from the sound region (reference region). The defects are arranged in the 4 by 4 array layout, and the distance between the centres of two adjacent holes is $31 \mathrm{~mm}$. As illustrated in Fig. 5(b), the holes were drilled with four groups of diameters $(5,10,15$, and $20 \mathrm{~mm})$ and four groups of thicknesses $(7,6,5$, and $4 \mathrm{~mm})$, representing defect depths of 1 , 2,3 , and $4 \mathrm{~mm}$, from the top inspection surface. The defects in each row have the same diameter but different depths, while the holes in each column have different diameters but the same depth. This sample is used to qualitatively and quantitatively evaluate the performance of the pulsed MAT system with comparative studies being taken up with the classic pulsed thermographic inspection using the high-resolution FLIR SC7600 IR camera.

The dimension of Sample 2 and Sample 3 is $150 \times 120 \times 5 \mathrm{~mm}^{\wedge} 3$, where impact damage is presented. Sample 2, shown in Fig. 6(a), contains a $42 \mathrm{~mm}$ length compound damage that features both crack and delamination. Sample 3, shown in Fig. 6(b), has a $22 \mathrm{~mm}$ "Zigzag" shaped crack. The inspection and evaluation of this type of complex damage is crucial to the life-assessment and maintenance of composite 
structures [33]. These two samples were used to qualitatively evaluate the performance of the laser MAT system with the classic laser thermographic inspection using the high-resolution FLIR A655sc IR camera.

\section{2) Experiments plan}

Two experiments were implemented for these three samples. For the comparison study, Experiment 1 was conducted on Sample 1 using the proposed MAT and SC7600 under the experimental setup shown in Fig. 2(a). A flash excitation source was used to provide homogenous heating for the front side of Sample 1. The working distance from the two cameras to the sample surface is $250 \mathrm{~mm}$. The flash energy applied to the sample was $2 \mathrm{~kJ}$. The framerate of Lepton and SC7600 was set at $8.7 \mathrm{~Hz}$ and $10 \mathrm{~Hz}$ respectively. The whole inspection duration is 70 seconds, indicating 609 and 700 frames being captured, respectively.

Experiment 2 was conducted on Sample 2 and 3 using the proposed laser MAT, shown in Fig. 2(b). The line laser beam was projected on the sample surface with a $45^{\circ}$ angle. The laser energy is $15 \mathrm{~W}$. The microbolometer based LWIR FLIR A655sc was used for the comparison purpose. The framerate of Lepton and A655sc was set at $8.7 \mathrm{~Hz}$ and $25 \mathrm{~Hz}$ respectively. Lepton was placed at $100 \mathrm{~mm}$ distance away from the sample surface while the working distance of A655sc was $200 \mathrm{~mm}$. An automatic XY stage facilitated the movement of the sample at a speed of $10 \mathrm{~mm} / \mathrm{sec}$. The sample was fixed on the XY stage horizontally, keeping the IR lens perpendicular to the sample surface. During the process of testing, the positions of the camera and laser head are fixed, while only the sample is moved using the XY stage. This setup aims to reduce the noise caused by the movement of the camera and the interference to the laser head.

\section{RESULT AND DISCUSSION}

\section{A. Results of the flash-based MAT system}

Fig. 7 plots the raw temperature-time decay curves in the logarithmic domain using the proposed MAT system and

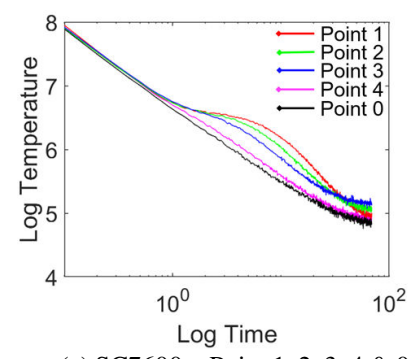

(a) SC7600---Point 1, 2, 3, 4 \& 0

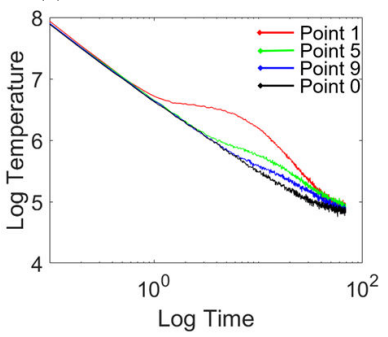

(b) SC7600---Point 1, 5, 9 \& 0

Fig. 7. Comparison of the temperature-time decay profiles in the logarithmic domain of the selected defects

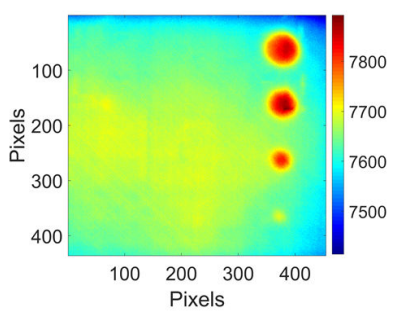

(a) SC7600---The No. 35 Frame

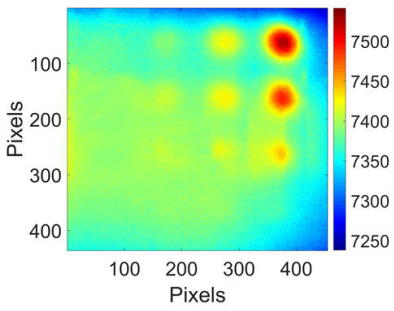

(b) SC7600----The No. 144 Frame

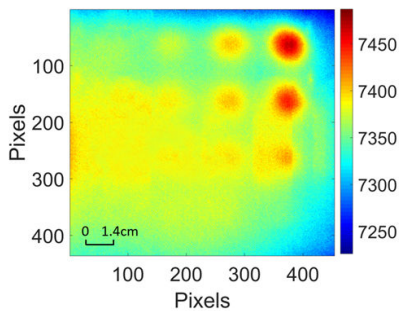

(c) SC7600---The No. 175 Frame

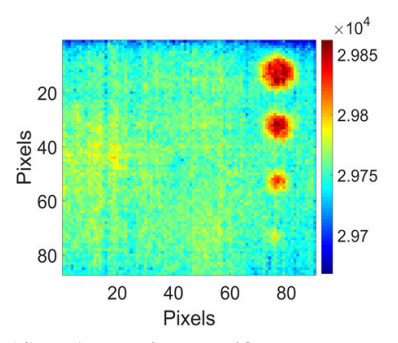

(d) MAT---The No. 49 Frame

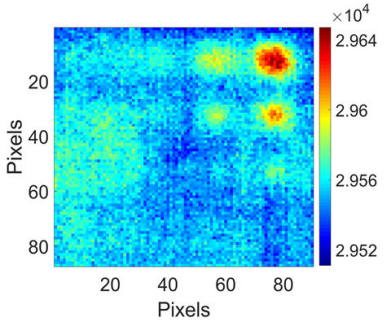

(e) MAT---The No. 145 Frame

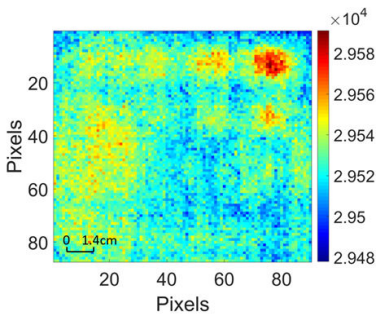

(f) MAT---The No. 186 Frame
Fig. 8. The raw thermal images at different frames for Sample 1

SC7600 system for the selected points. Fig. 7(a) and (c) show the curves representing defects with the same depth but different sizes, as well as the reference. Fig. 7(b) and (d) show the curves for defects with the same size at different depths. It can be observed from the comparison that although SC7600 has better signal quality than the Lepton sensor, the MAT system can effectively capture the difference of decay curve among the selected defects and the reference for both groups. This observation suggests that the proposed MAT system can detect the selected defects with reduced contrast.

Due to the difference of defect size and depth, the maximal temperature contrast to sound areas for each defect appears at different times. Fig. 8(a)-(c) show the $35^{\text {th }}, 144^{\text {th }}$ and $175^{\text {th }}$ thermal images post flash from SC7600 and Fig. 8(d)-(f) show the $49^{\text {th }}, 145^{\text {th }}$ and $186^{\text {th }}$ frames after the flash from the proposed MAT system. It should be noted that the framerate is different between the two systems and therefore the frame showing the highest contrast is different. Results from both systems show that the defects with shallow depth, such as Defect 1-4, appear earlier. In addition, relatively sharp edges can be observed on the large defects, while the results of smaller ones have blurred edges. Not all defects can be detected due to a small radius/depth ratio for both systems [34]. It should be noted that Fig. 8 uses a global colourmap and therefore the contrast of small or deep defects is not as sharp as that of large and shallow defects. To better evaluate the detectability, a region of $100 \times 100$ pixels for each defect is selected for the SC7600 and a region of $25 \times 25$ pixels for each defect is selected for the MAT for comparison and the results are as tabulated in Table I. The images presented in the table shows the highest contract frame 
TABLE I. VISUALISATION OF EACH DEFECT USING A LOCAL CONTRAST COLORMAP FOR THE PROPOSED SYSTEM AND SC7600

\begin{tabular}{|c|c|c|c|c|c|c|c|}
\hline Defect & $\begin{array}{l}\text { Radius } \\
\text { (mm) }\end{array}$ & $\begin{array}{l}\text { Depth } \\
(\mathrm{mm})\end{array}$ & \begin{tabular}{|c|} 
Radius/Dept \\
h ratio
\end{tabular} & SC760 & & MAT & \\
\hline 1 & 10 & 1 & 10 & 8 & $\checkmark$ & & $\checkmark$ \\
\hline 2 & 7.5 & 1 & 7.5 & 0 & $\checkmark$ & & $\checkmark$ \\
\hline 3 & 5 & 1 & 5 & 0 & $\checkmark$ & & $\checkmark$ \\
\hline 4 & 2.5 & 1 & 2.5 & C & $\checkmark$ & & $\checkmark$ \\
\hline 5 & 10 & 2 & 5 & & $\checkmark$ & & $\checkmark$ \\
\hline 6 & 7.5 & 2 & 3.75 & (0) & $\checkmark$ & & $\checkmark$ \\
\hline 7 & 5 & 2 & 2.5 & 10 & $\checkmark$ & & $\checkmark$ \\
\hline 8 & 2.5 & 2 & 1.25 & * & $\checkmark$ & 43 & \\
\hline 9 & 10 & 3 & 3.33 & e & $\checkmark$ & 80 & $\checkmark$ \\
\hline 10 & 7.5 & 3 & 2.5 & & $\checkmark$ & & $\checkmark$ \\
\hline 11 & 5 & 3 & 1.67 & & $\checkmark$ & & \\
\hline 12 & 2.5 & 3 & 0.83 & & & & \\
\hline 13 & 10 & 4 & 2.5 & & & s. & \\
\hline 14 & 7.5 & 4 & 1.88 & & & & \\
\hline 15 & 5 & 4 & 1.25 & 20 & & 32 & \\
\hline 16 & 2.5 & 4 & 0.63 & 4. & & $y^{2}$ & \\
\hline
\end{tabular}

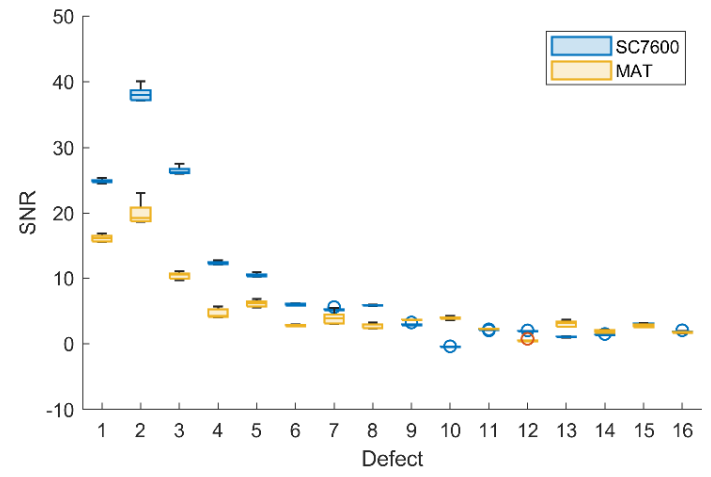

Fig. 9. Comparison of SNR for SC7600 and MAT system for each defect

of the first derivative of TSR using a local colourmap. The images from the MAT system are scaled up 4 times with a media filter for easier comparison. It can be observed that

- The SC7600 can detect 11 defects including Defect 1, 2, 3, $4,5,6,7,8,9,10,11$ while MAT can detect 9 defects including Defect 1, 2, 3, 4, 5, 6, 7, 9, 10. For the SC7600, the threshold of the detectable radius/depth ratio is 1.25 and the threshold of the detectable depth is $3 \mathrm{~mm}$. Defect 13 and 14 are not well represented even though the radius/depth ratio is over 1.25.

- For the proposed MAT system, the threshold of the detectable radius/depth ratio is 2.5 and the threshold of the detectable depth is $3 \mathrm{~mm}$.

To quantify the detectability of each defect, the SNR values, in our case to be the contrast-to-noise ratio, for all 16 defects were calculated for each frame using the following equation [35]
TABLE II. THE ESTIMATED DEPTH AND GROUND TRUTH OF THE SELECTED DEFECTS FOR SAMPLE 1

\begin{tabular}{|c|c|c|c|c|c|c|c|}
\hline \multicolumn{3}{|c|}{ Point No. } & 1 & 2 & 3 & 5 & 6 \\
\hline \multicolumn{3}{|c|}{ Ground Truth (mm) } & 1 & 1 & 1 & 2 & 2 \\
\hline \multirow{6}{*}{ SC7600 } & \multirow{6}{*}{$\begin{array}{c}\text { Estimated } \\
\text { Depth } \\
(\mathrm{mm})\end{array}$} & LSD & 1.05 & 0.97 & 0.89 & 1.90 & 1.86 \\
\hline & & Error & 0.05 & 0.03 & 0.11 & 0.10 & 0.14 \\
\hline & & $\%$ & 5.0 & 3.0 & 11.0 & 5.0 & 7.0 \\
\hline & & NLSF & 1.01 & 0.98 & 0.90 & 2.09 & 1.97 \\
\hline & & Error & 0.01 & 0.02 & 0.10 & 0.09 & 0.03 \\
\hline & & $\%$ & 1.0 & 2.0 & 10.0 & 4.5 & 1.5 \\
\hline \multirow{6}{*}{ MAT } & \multirow{6}{*}{$\begin{array}{c}\text { Estimated } \\
\text { Depth } \\
(\mathrm{mm})\end{array}$} & LSD & 1.12 & 1.19 & 1.25 & 1.87 & 1.82 \\
\hline & & Error & 0.12 & 0.19 & 0.25 & 0.13 & 0.18 \\
\hline & & $\%$ & 12.0 & 19.0 & 25.0 & 6.5 & 9.0 \\
\hline & & NLSF & 1.05 & 0.98 & 0.89 & 2.10 & 2.09 \\
\hline & & Error & 0.05 & 0.02 & 0.11 & 0.10 & 0.09 \\
\hline & & $\%$ & 5.0 & 2.0 & 11.0 & 5.0 & 4.5 \\
\hline
\end{tabular}

where $\overline{T_{d}(t)}$ is the average temperature over a defect region $\left(5 \times 5\right.$ pixels for the SC7600 and $3 \times 3$ pixels for the MAT), $\overline{T_{s}(t)}$ is the average temperature over a sound region $(20 \times 20$ pixels for the SC7600 and 5×5 pixels for the MAT). The SNR values for a total of 200 frames of each defect were calculated and the top $10 \%$ was selected to represent the detectability, the results of which are shown in Fig. 9. As expected, the SNR values of the SC7600 for most defects are consistently higher than the MAT system, representing a superior detectability. For the defects with a large radius/depth ratio, such as Defect 1-5, the SNR for the MAT is large than 5, indicating a good detectability. Detailed SNR values for each frame can be found in the Supplementary Materials. All the above observations suggest that although the detectability of MAT is compromised due to the low specification of temperature and spatial resolutions, the MAT can be effectively used to detect defects in composites. More detailed results can be found in the Supplementary Materials.

To further quantitatively evaluate the performance, Table II shows the estimated defect depths of the selected points using the LSD and NLSF methods for two systems. For the same defect, the SC7600 measures the depth more accurately (error up to $11 \%$ ) than the MAT (error up to $25 \%$ ). In terms of two

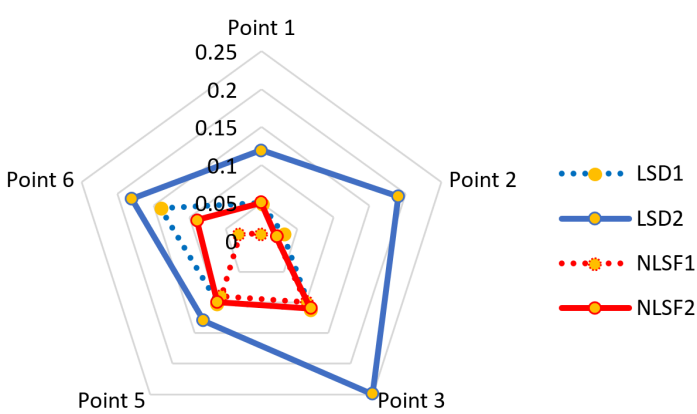

Fig. 10. The error range of SC7600 and the MAT system using LSD and NLSF methods, where LSD1 and NLSF1 come from SC7600 (dash line), and LSD2 and NLSF2 come from MAT (solid line). 


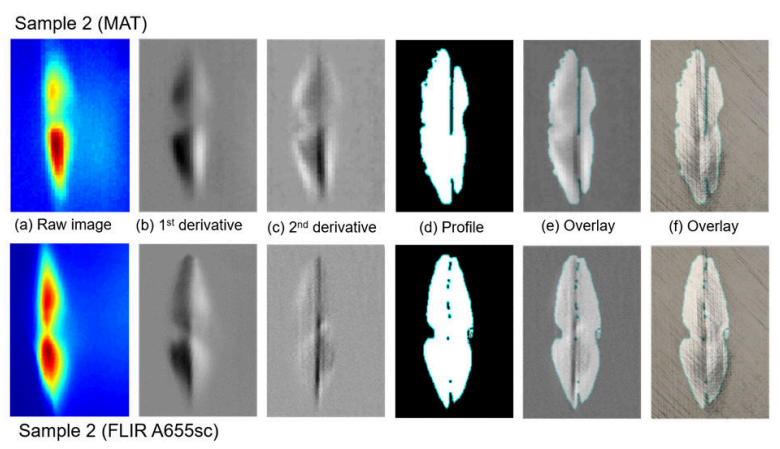

Fig. 11. Damage visualisation using the laser MAT system (top row) and A655sc (bottom row) for Sample 2

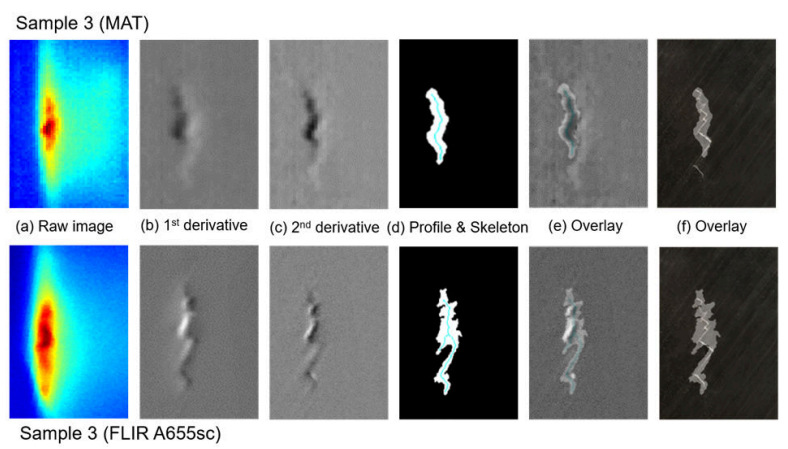

Fig 12. Damage visualisation using the laser MAT system (top row) and A655sc (bottom row) for Sample 3

depth measurement methods, the NLSF (average errors are $3.8 \%$ and $5.5 \%$ for two systems respectively) performs better than LSD (average errors are $6.2 \%$ and $14.3 \%$ for two systems respectively). It is observed that NLSF works particularly better than LSD for the MAT where the noise level is high, which confirms the research finding in [28]. This is because NLSF is based on fitting a physical model while LSD is based on fitting a numerical model where the model can be easily over-fitted when the noise level is high. Although the high-resolution camera is superior to the MAT system in the overall depth measurement accuracy, there is no significant difference (e.g. max error $10 \%$ vs $11 \%$ ) if the right method is chosen. Fig. 10 plots a polygon map of depth measurement error using the LSD and the NLSF for Sample 1. It can be observed that although the SC7600 with flash excitation overall performs better than pulsed MAT for both algorithms, the performance of NLSF based on MAT is even better than that of LSD with SC7600.

\section{B. Results of the laser-based MAT system}

Fig. 11 and 12 show the detection results for Samples 2 and 3 using the proposed MAT system and the A655sc based on the laser excitation, where six visualisations are presented including the raw images, the $1^{\text {st }}$ derivative, the $2^{\text {nd }}$ derivative, the extracted profile of the defect, the overlay of profile and the $2^{\text {nd }}$ derivative image, and the overlay of profile and the digital surface image (from left to right). In the raw images, the laser beam overlaps with the damage which makes it a challenge to see the true damage profile. After applying the Savitzky-Golay smoothing filter, the first and the second TSR derivatives of the filtered IR images (see (b) and (c) graphs) improve the damage

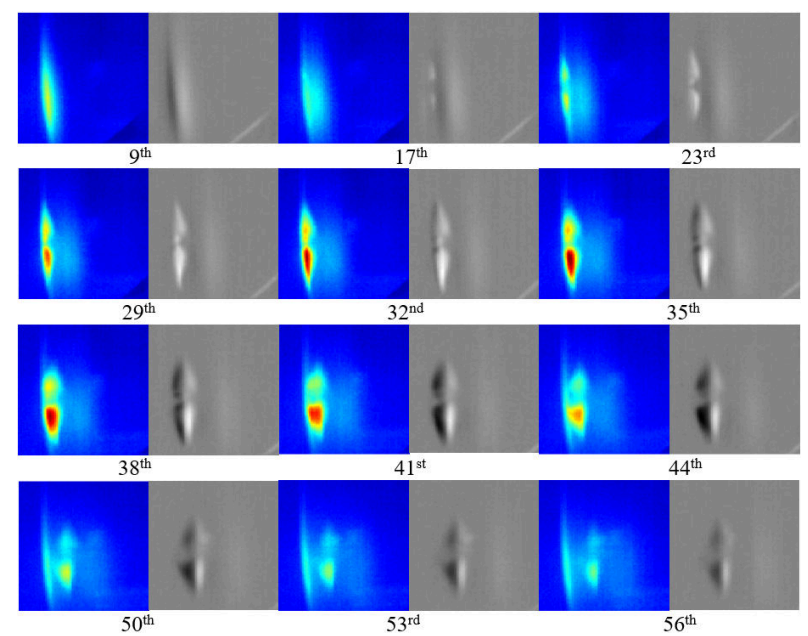

Fig. 13. The raw thermal images (colour) and corresponding 1st derivative (gray) for different frames during the scan of the proposed laser MAT system for Sample 2.
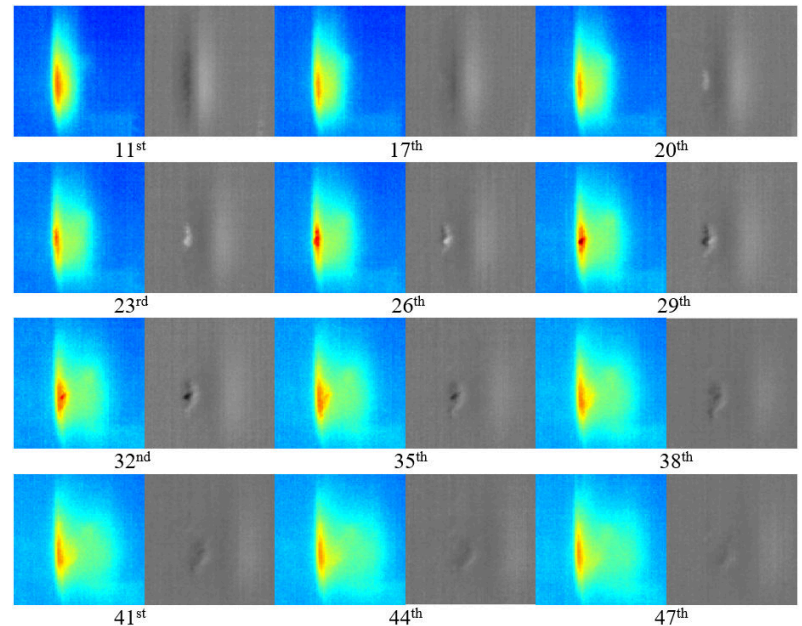

Fig. 14. The raw thermal images (colour) and corresponding 2 nd derivative (gray) for different frames during the scan of the proposed laser MAT system for Sample 3.

representation with a high-contrast contour, after the removal of the laser beam from the images. For Sample 2, a butterfly shape impact damage can be well observed in the $1^{\text {st }}$ derivative image. The vertical crack, shown in Fig. 6(a), can be observed in the $2^{\text {nd }}$ derivative image. For Sample 3 , the zigzag crack can be observed in both $1^{\text {st }}$ and $2^{\text {nd }}$ derivative images while the $2^{\text {nd }}$ derivative image shows sharper edges. The results from the MAT system have less sharp boundary than those from A655sc mainly due to the lower spatial resolution. However, it does not affect the determination of the type, profile and measurement of damage. In addition, the $2^{\text {nd }}$ derivative is more appropriate to detect sharp damage (e.g. crack) and the $1^{\text {st }}$ derivative is more appropriate to detect damage with a large area (e.g. delamination).

To further understand how to select the optimised frame to represent the defects of Sample 2 and 3, Fig. 13 and 14 were produced to show the raw thermal image and corresponding visualisation of the detected defect at different frames. The first row shows three frames when the laser line is on the right of the defect, where the defect can barely be observed. The second row shows three frames when the laser line is overlapping with 
the defect, where a partial defect is visible. The third row shows three frames when the laser line is on the left of the defect, which offer the optimised representation of the defect with high contrast. When the laser line passes the defect for a while, as shown in the fourth row, the contrast of defect decreases.

All these observations demonstrate that the proposed laser MAT can effectively evaluate impact damage of composite laminates, particularly for large delamination, the performance is similar to the classic laser thermography.

\section{CONCLUSION}

Aiming to improve the applicability and accessibility of active thermography and simultaneously reduce the cost of the inspection equipment, a novel MAT system including hardware integration and algorithm development was proposed in this paper with its qualitative and quantitative evaluation to detect defects and damage in composite laminates. Integrated with Raspberry $\mathrm{Pi}$ and two different exciting sources, the performance of the proposed MAT system has been to detect flat bottom holes, impact damage and crack in composite laminates, in comparison to existing systems with high-end infrared sensors. The key findings from this study include:

- The low-cost and miniaturised IR sensor introduces more noise than the SC7600. For Sample 1 with 16 flat-bottom holes, the average SNR is $10 \mathrm{~dB}$ lower.

- Although the detectability of defects against radius/depth ratio is compromised due to the increased noise level and reduced spatial resolution, the proposed MAT system has the most capability of the high-end system in terms of damage detection and depth measurement in CFRP laminates.

- For the developed system, depth measurement methods based on fitting a physical model are recommended over the models based on fitting a numerical model due to their superior performance against the high-level noise.

- For the laser-based MAT system, the $1^{\text {st }}$ and the $2^{\text {nd }}$ derivative TSR images show improved performance in representing the damage than the raw images. The $1^{\text {st }}$ derivative image is more appropriate for damages with a large size while the $2^{\text {nd }}$ derivative image is more appropriate for damages with a sharp boundary.

- Data pre-processing methods, such a TSR, Savitzky-Golay smoothing filter and media filter are strongly recommended to reduce temporal and spatial noise before quantitative analysis.

The contribution of this paper from the scientific point of view includes: (1) The current miniaturised IR cameras feature low-resolution and low SNR, which leads to the poor performance of most of current data analysis methods. We propose an effective data process, image processing and feature extraction method to reduce the influence of noise and enhance the detectability of damage; (2) For the flash MAT system, this paper discussed how to measure the defect depth more accurately; (3) For the laser MAT system working under the inline scan mode, this paper presented how to select the optimal frames to best represent damage.

One limitation of this system is that it has a sample rate of fewer than $10 \mathrm{fps}$, which limits its application to capture the thermal behaviour of materials with high thermal conductivity and diffusivity. The proposed system has a prominent price advantage and significantly small volume. Its small and flexible body could better adapt to complex and geometrically intricate space in the industrial in-situ inspection.

\section{REFERENCES}

[1] D. Axinte et al., "MiRoR-Miniaturized Robotic Systems for Holistic In-Situ Repair and Maintenance Works in Restrained and Hazardous Environments," IEEE/ASME Trans. Mechatronics, vol. 23, no. 2, pp. 978-981, Apr. 2018, doi: 10.1109/TMECH.2018.2800285.

[2] X. Dong et al., "Development of a slender continuum robotic system for on-wing inspection/repair of gas turbine engines," Robot. Comput. Integr. Manuf., vol. 44, pp. 218-229, Apr. 2017, doi: 10.1016/j.rcim.2016.09.004.

[3] Z. Chen, Z. Jiang, W. Gui, and C. Yang, "A Novel Device for Optical Imaging of Blast Furnace Burden Surface: Parallel Low-Light-Loss Backlight High-Temperature Industrial Endoscope," IEEE Sens. J., vol. 16, no. 17 , pp. 6703-6717, Sep. 2016, doi: 10.1109/JSEN.2016.2587729.

[4] Karl Storz, "Flexible Endoscopes Extremely flexible endoscopes." https://www.karlstorzndtec.com/fileadmin/user_upload/produkte/Produ ktdatenblaetter/96191077_KSNDTec_PB3_Produktbrosch-E_15-012019.pdf.

[5] M. J. Lovejoy, Magnetic Particle Inspection: A practical guide. Springer Science \& Business Media, 2012.

[6] Johnson \& Allen, "Permanent Magnet." https://www.johnsonandallen.co.uk/product/rpns.

[7] A. du Plessis, S. G. le Roux, and A. Guelpa, "The CT Scanner Facility at Stellenbosch University: An open access X-ray computed tomography laboratory," Nucl. Instruments Methods Phys. Res. Sect. B Beam Interact. with Mater. Atoms, vol. 384, pp. 42-49, Oct. 2016, doi: 10.1016/j.nimb.2016.08.005.

[8] U. Ewert, "Current Developments in Digital Radiography and Computed Tomography from nm to Macro Scale," in 12th European Conference on Non-Destructive Testing (ECNDT 2018)., 2018, pp. 1-9.

[9] General Electric, "DXR250C-W/DXR250U-W Wireless Digital X-ray Detector." https://www.gemeasurement.com/inspectionndt/radiography-and-computed-tomography/dxr250c-wdxr250u-wdigital-detectors.

[10] F. Huan, L. Xiucheng, W. Bin, H. Qiang, and H. Cunfu, "Design of a miniaturised ultrasonic guided wave inspection instrument for steel strand flaw detection," Insight - Non-Destructive Test. Cond. Monit., vol. 59, no. 1, pp. 17-23, Jan. 2017, doi: 10.1784/insi.2017.59.1.17.

[11] Sonotron NDT, "ISONIC utPod." http://www.sonotronndt.com/utpod.htm.

[12] W. Du, Y. Zhao, R. Roy, S. Addepalli, and L. Tinsley, "A review of miniaturised Non-Destructive Testing technologies for in-situ inspections," Procedia Manuf., vol. 16, no. November, pp. 16-23, 2018, doi: 10.1016/j.promfg.2018.10.152.

[13] D. Thapa, N. Samadi, A. Parkhimchyk, and N. Tabatabaei, "Comparison of low cost and research grade active thermography platforms for detection of early dental caries," in Optical Sensing and Detection VI, Apr. 2020, no. May, p. 10, doi: 10.1117/12.2555279.

[14] Automation Technology, "C-CheckIR." https://www.automationtechnology.de/cms/en/mobile-ndt-systems-formaintenance/.

[15] W. Du, H. Liu, A. Sirikham, S. Addepalli, and Y. Zhao, "A miniaturised active thermography system for in-situ inspections," in 4th IFAC Workshop on Advanced Maintenance Engineering, Service and Technology, September 10-11, 2020. Cambridge, UK.

[16] R. Fuente et al., "Development and demonstration of an automated system for limited access weld inspection by using infrared active thermography," in 7th International Symposium on NDT in Aerospace 16- 18 Nov 2015, Bremen, Germany (AeroNDT 2015)

[17] W. Du, S. Addepalli, and Y. Zhao, "The Spatial Resolution Enhancement for a Thermogram Enabled by Controlled Sub-pixel Movements," IEEE Trans. Instrum. Meas., pp. 1-1, 2019, doi 10.1109/TIM.2019.2932175.

[18] C. Ibarra-Castanedo et al., "Comparative Study of Active Thermography Techniques for the Nondestructive Evaluation of Honeycomb Structures," Res. Nondestruct. Eval., vol. 20, no. 1, pp. 1- 
31, Jan. 2009, doi: 10.1080/09349840802366617.

[19] X. Lu et al., "Pulsed Air-Flow Thermography for Natural Crack Detection and Evaluation," IEEE Sens. J., vol. 20, no. 14, pp. 80918097, 2020, doi: 10.1109/JSEN.2020.2982556.

[20] L. Lei, G. Ferrarini, A. Bortolin, G. Cadelano, P. Bison, and X. Maldague, "Thermography is cool: Defect detection using liquid nitrogen as a stimulus," NDT E Int., vol. 102, no. May 2018, pp. 137143, Mar. 2019, doi: 10.1016/j.ndteint.2018.11.012.

[21] X. Zhang et al., "CFRP Impact Damage Inspection Based on Manifold Learning Using Ultrasonic Induced Thermography," IEEE Trans. Ind. Informatics, vol. 15, no. 5, pp. 2648-2659, May 2019, doi: 10.1109/TII.2018.2866413.

[22] B. Gao, L. Bai, W. L. Woo, G. Y. Tian, and Y. Cheng, "Automatic Defect Identification of Eddy Current Pulsed Thermography Using Single Channel Blind Source Separation," IEEE Trans. Instrum. Meas., vol. 63, no. 4, pp. 913-922, Apr. 2014, doi: 10.1109/TIM.2013.2285789.

[23] C. Pei, H. Liu, J. Qiu, T. Liu, and Z. Chen, "Progress on the ultrasonic testing and laser thermography techniques for NDT of tokamak plasmafacing components," Theor. Appl. Mech. Lett., vol. 9, no. 3, pp. 180187, May 2019, doi: 10.1016/j.taml.2019.03.011.

[24] A. Sirikham, Y. Zhao, H. Y. Nezhad, W. Du, and R. Roy, "Estimation of Damage Thickness in Fiber-Reinforced Composites using Pulsed Thermography," IEEE Trans. Ind. Informatics, vol. 15, no. 1, pp. 445453, Jan. 2019, doi: 10.1109/TII.2018.2878758.

[25] J. Zhu, G. Tiany, Q. Min, and J. Wu, "Comparison Study of Different Features for Pocket Length Quantification of Angular Defects Using Eddy Current Pulsed Thermography," IEEE Trans. Instrum. Meas., vol. 68, no. 5, pp. 1373-1381, May 2019, doi: 10.1109/TIM.2018.2890053.

[26] S. K. Lau, D. P. Almond, and J. M. Milne, "A quantitative analysis of pulsed video thermography," NDT E Int., vol. 24, no. 4, pp. 195-202, 1991, doi: 10.1016/0963-8695(91)90267-7.

[27] S. M. Shepard, "Reconstruction and enhancement of active thermographic image sequences," Opt. Eng., vol. 42, no. 5, p. 1337 , May 2003, doi: 10.1117/1.1566969.

[28] A. Sirikham, Y. Zhao, and J. Mehnen, "Determination of thermal wave reflection coefficient to better estimate defect depth using pulsed thermography," Infrared Phys. Technol., vol. 86, pp. 1-10, Nov. 2017, doi: 10.1016/j.infrared.2017.08.012.

[29] D. L. Balageas, J.-M. Roche, F.-H. Leroy, W.-M. Liu, and A. M. Gorbach, "The thermographic signal reconstruction method: A powerful tool for the enhancement of transient thermographic images," Biocybern. Biomed. Eng., vol. 35, no. 1, pp. 1-9, 2015, doi: 10.1016/j.bbe.2014.07.002.

[30] J. G. Sun, "Method for Determining Defect Depth using Thermal Imaging," US6542849B2, 2003.

[31] A. Savitzky and M. J. E. Golay, "Smoothing and Differentiation of Data by Simplified Least Squares Procedures.," Anal. Chem., vol. 36, no. 8, pp. 1627-1639, Jul. 1964, doi: 10.1021/ac60214a047.

[32] Y. Zhao, S. Addepalli, A. Sirikham, and R. Roy, "A confidence map based damage assessment approach using pulsed thermographic inspection," NDT E Int., vol. 93, no. September 2017, pp. 86-97, Jan. 2018, doi: 10.1016/j.ndteint.2017.10.001.

[33] B. Wang, S. Zhong, T.-L. Lee, K. S. Fancey, and J. Mi, "Nondestructive testing and evaluation of composite materials/structures: A state-of-the-art review," Adv. Mech. Eng., vol. 12, no. 4, pp. 1-28, Apr. 2020, doi: $10.1177 / 1687814020913761$

[34] M. F. Beemer and S. M. Shepard, "Aspect ratio considerations for flat bottom hole defects in active thermography," Quant. Infrared Thermogr. J., vol. 15, no. 1, pp. 1-16, Jan. 2018, doi: $10.1080 / 17686733.2017 .1328642$

[35] G. Silipigni et al., "Optimization of the pulse-compression technique applied to the infrared thermography nondestructive evaluation," $N D T$ E Int., vol. 87, no. August 2016, pp. 100-110, 2017, doi: 10.1016/j.ndteint.2017.01.011. 
2020-10-13

\title{
A miniaturised active thermography system to inspect composite laminates
}

\author{
Du, Weixiang
}

IEEE

Du W, Liu H, Zhao Y, et al., (2020) A miniaturised active thermography system to inspect composite laminates. IEEE Transactions on Industrial Informatics, Available online 13 October 2020 https://doi.org/ 10.1109/TII.2020.3030619

Downloaded from Cranfield Library Services E-Repository 